\title{
Spectropolarimetry as a tool for understanding the diversity of planetary atmospheres
}

\section{Rossi ${ }^{1}$ (D) - J. Berzosa-Molina ${ }^{2}$ - J.-M. Desert ${ }^{3}$ - L. Fossati ${ }^{4} \cdot$ A. García Muñoz ${ }^{5}$. C. Haswell ${ }^{6}$ P. Kabath ${ }^{7}$. K. Kislyakova ${ }^{8}$. D. Stam ${ }^{9}$ (D) . A. Vidotto ${ }^{10}$}

Received: 1 September 2020 / Accepted: 18 October 2021 / Published online: 11 December 2021 (C) The Author(s) 2021

\begin{abstract}
The polarization state of starlight reflected by a planetary atmosphere uniquely reveals coverage, particle size, and composition of aerosols as well as changing cloud patterns. It is not possible to obtain a comparable level of detail from flux-only observations. It is therefore a powerful tool to better understand the crucial role played by clouds and aerosols in the chemistry, dynamics, and radiative balance of a planet. Furthermore, polarization observations can probe the atmosphere of planets independently of the orbital geometry (hence it applies to both transiting and non-transiting exoplanets). A high-resolution spectropolarimeter with a broad wavelength coverage, particularly if attached to a large space telescope, would enable simultaneous study of the polarimetric planetary properties of the continuum and to look for and characterize the polarimetric signal due to scattering from single molecules, providing detailed information about the composition and vertical structure of the atmosphere.
\end{abstract}

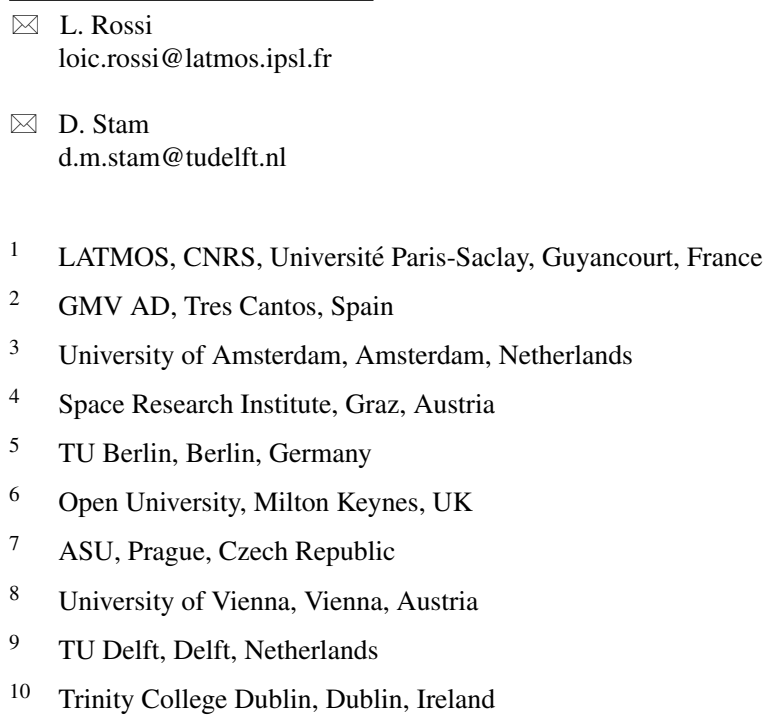


Keywords Atmospheres · Exoplanets · Polarimetry

\section{Introduction}

The discovery of exoplanets opened an exciting new field in astrophysics and planetary sciences. Almost 4000 exoplanets are currently known and thousands more are expected to be found in the near future. As a natural consequence of this success, the research focus is shifting from planet detection to atmospheric characterization, as shown, e.g., by the fact that TESS and PLATO focus on searching for transiting planets orbiting nearby, bright stars, specifically to enable atmospheric characterization. Despite the still limited sample and data quality, observations clearly indicate that exoplanetary atmospheres are extremely diverse, ranging from planets showing strong atomic and molecular features to others which are nearly featureless, indicating the possible presence of aerosols (e.g., [26]). These data are mostly obtained through transmission spectroscopy, which however provides no or very limited information on the microphysical properties (particle composition, size, shape, number density) of cloud and haze layers. In our Solar System, many questions remain to be answered regarding planetary atmospheres, in particular about the formation, evolution, and effect of clouds and aerosols on the global circulation, the chemistry, and the radiative balance. Broad wavelength coverage, high-resolution spectropolarimetry of starlight that is reflected by planets promises to be a game changer in our quest towards better understanding the atmospheres of these alien worlds, particularly if carried out simultaneously with total flux measurements.

\section{Spectropolarimetry of planetary atmospheres}

\subsection{General considerations}

Polarimetry is a powerful tool for studying (exo)planets. Since reflections in general polarize light, the starlight reflected by a planet is polarized whereas, integrated over the stellar disc, the light of most main-sequence stars is unpolarized $[3,4,16]$. The degree of linear polarization ( $\mathrm{P}$; the polarized-to-total flux ratio) is sensitive to the properties of the scattering particles, the atmospheric structure, and, if present, the reflection by the planetary surface. It is however relatively insensitive to a number of instrumental effects, and can be determined with high accuracy $\left(\simeq 10^{-5}-10^{-6}\right)$. Several instruments looking for long-period exoplanets through direct imaging (e.g., SPHERE, GPI) employ polarization to increase the star-planet contrast and facilitate planet detection. Polarimetry is considered for good reasons to be a primary tool for detecting water and oxygen on directly imaged exoplanets (e.g., [1, 5, 15, 18, 19])

The line and continuum polarization state of starlight that is reflected by a planet depends on the star-planet-observer phase angle (i.e., the angle between the star and the observer as seen from the center of the planet) and is sensitive to the optical properties of the planetary atmosphere and surface (Fig 1; e.g., [20, 24, 29-31]) 
The degree of linear polarization of a celestial body can reach several tens of percent (e.g., Titan), in particular when the planetary phase angle is close to $90^{\circ}$ (i.e., quadrature; [12, 23, 24, 27]).

However, for spatially unresolved exoplanets, $\mathrm{P}$ will depend strongly on the stellar background signal, which adds unpolarized light to the planetary signal hampering polarization measurements. At phase angles close to $0^{\circ}$ or $180^{\circ}, \mathrm{P}$ is close to zero (Fig. 1). Thus, polarimetry is also ideally suited to characterize the atmospheres of non-transiting exoplanets.

\subsection{Sources of polarization}

The degree and direction of polarization of the starlight scattered and reflected by a planet depends on the illumination, viewing geometry, optical properties of the atmospheric constituents, reflection properties of the planetary surface, and wavelength. Several processes can polarize light in planetary atmospheres: Rayleigh diffusion, hazes, aerosols, etc.

The Rayleigh scattering cross section varies roughly as $1 / \lambda^{4}$, making $\mathrm{P}$ larger at shorter wavelengths, while scattering by larger aerosols varies in a more complex way, and the transition between the two processes depends on the aerosol's microphysical properties, their distribution in the atmosphere and atmospheric pressure.

The polarization phase function of a planet carries the characteristic signatures of the light that is singly scattered by gas, aerosol, and/or cloud, and these, unlike for the total flux, are not erased by multiple scattering. Therefore, polarization observations are compelling for advancing our understanding of planetary atmospheres.

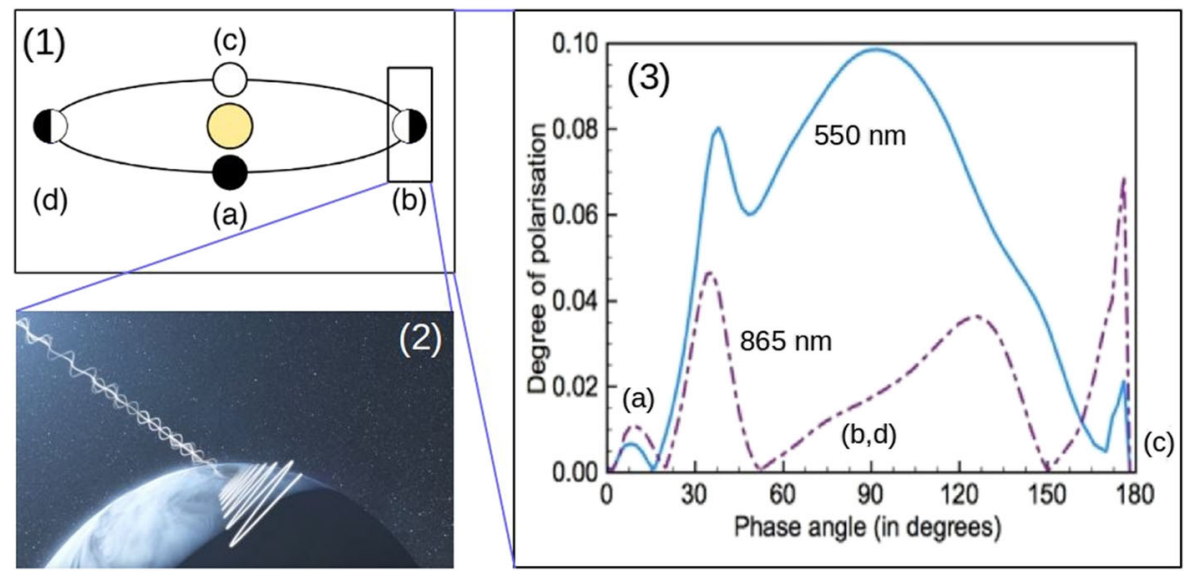

Fig. 1 Panels (1) \& (2): schematic of a planetary system in which the unpolarized starlight becomes polarized through reflection by a planetary atmosphere. Panel (3): degree of polarization (P; in \%) of a cloudy Earth-like planet as a function of planetary orbital phase, labeled as in panel (1). The different lines are for different wavelengths. Credits: (1) S. Wiktorowicz (2) ESO/L. Calçada/Nick Risinger (skysurvey.org) (3) D. M. Stam 


\subsection{Microphysical properties}

In particular, polarization is sensitive to the microphysical properties of planetary atmospheres. Polarization allows one to derive size and shape of the particles forming the clouds; for example, the single scattering properties of ice (crystal) particles are usually very different from those of liquid (spherical) particles (e.g., [9]). Features like glories and rainbows can be detected in the polarization phase curves at short phase angles. Detection of such phenomena provide immediate information about the shape and refractive index of the droplets that generated them $[1,15,17,21]$.

This is critical information for understanding how clouds develop and evolve. The sensitivity of polarization to the microphysical properties of the scatterers in a planetary atmosphere make this a key tool for breaking degeneracies which can arise for flux-only observations. For example, [10] and [32] used disc-integrated polarimetry of Venus and Titan, respectively, to derive the composition and size of the cloud particles. Due to the lack of sensitivity, total flux measurements cannot provide this information.

\subsection{Dependence on planetary parameters}

Polarimetry can provide information about the vertical and horizontal structure of an atmosphere and even about orbital parameters.

Figure 2 shows the strong dependence of the polarization of an Earth-like planet upon surface albedo and cloud coverage, which play a key role in the energy budget of a planetary atmosphere and in turn influence atmospheric composition (e.g., [13, $14,18])$. As a vectorial quantity, the polarization of an exoplanet will depend on the inclination of the orbit with respect to the observer (cf. Fig. 2, right panel), providing information about this parameter that is difficult to obtain only using flux.

Furthermore, detecting irregular temporal variations in polarization could indicate changing cloud patterns (i.e., weather), constraining also heat transportation and distribution (e.g., [7, 19]).

For example, polarimetry has enabled the identification of seasonal variations on Jupiter. The level of polarization that has been measured at the poles of Jupiter in the
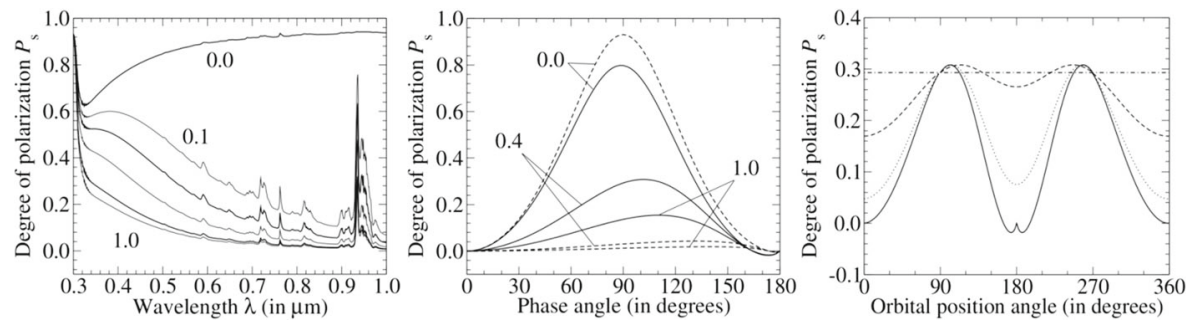

Fig. 2 Left: degree of polarization $(\mathrm{P})$ of starlight reflected by model planets with Earth-like clear atmospheres vs wavelength and surface albedo (0.0 to 1.0). Middle: $\mathrm{P}$ vs phase angle for surface albedos of 0.0 , 0.4 , and 1.0 and at wavelengths of 0.44 (solid) and $0.87 \mu \mathrm{m}$ (dashed). Right: P vs orbital position angle at a wavelength of $0.44 \mu \mathrm{m}$ and for a surface albedo of 0.4 , for orbital inclination angles of $0^{\circ}$ (dot-dashed line), $30^{\circ}$ (dashed line), $60^{\circ}$ (dotted line), and $90^{\circ}$ (solid line). From Stam (2008) 
optical band goes up to a few percent, but one pole generally presents a higher value than the other. [25] identified variations in this difference between the level of polarization at the poles, identifying a further correlation with the solar longitude. This difference is believed to be connected with an increase of hazes at the less irradiated (winter) pole.

\subsection{Polarimetry in the ultraviolet}

Since polarization simulations of starlight reflected by an exoplanet extending to the ultraviolet (UV), where $\mathrm{P}$ is believed to be particularly large, have never been performed before, we employed the PyMieDAP code [22] to show the exquisite sensitivity of UV polarization measurements to the physical properties of planetary atmospheres. Figure 3 shows flux (top row) and P (bottom row) as a function of phase angle at three UV wavelengths $(150,200$, and $300 \mathrm{~nm})$ for a HD189733b-like exoplanet hosting a clear atmosphere (left) and an atmosphere dominated by $\mathrm{NH}_{3}$ (middle) or $\mathrm{MgSiO}_{3}$ (enstatite; right) aerosols. The three curves have very distinct behaviors, particularly as a function of wavelength, clearly indicating the potential of UV spectropolarimetry for gaining critical information on planetary atmospheres, complementary to that provided by flux-only measurements.

Previous efforts have concentrated on low spectral resolution $(<100)$ instruments trying to drive up the signal-to-noise ratio. A major difficulty of broadband polarimetry is the need to correct for spurious signals introduced by the interstellar medium, the Earth's atmosphere, or the telescope. It has been recently shown that high resolution $(>100,000)$ spectropolarimetric observations are possible and are correspondingly powerful in constraining atmospheric properties [8]. The polarization of a spatially-unresolved exoplanet can be detected by cross-correlating high-dispersion linear polarization and intensity spectra of the star-planet system. This technique allows one to separate polarization contributions originated at different radial velocities that might be proved to be useful for disentangling the planet's polarization
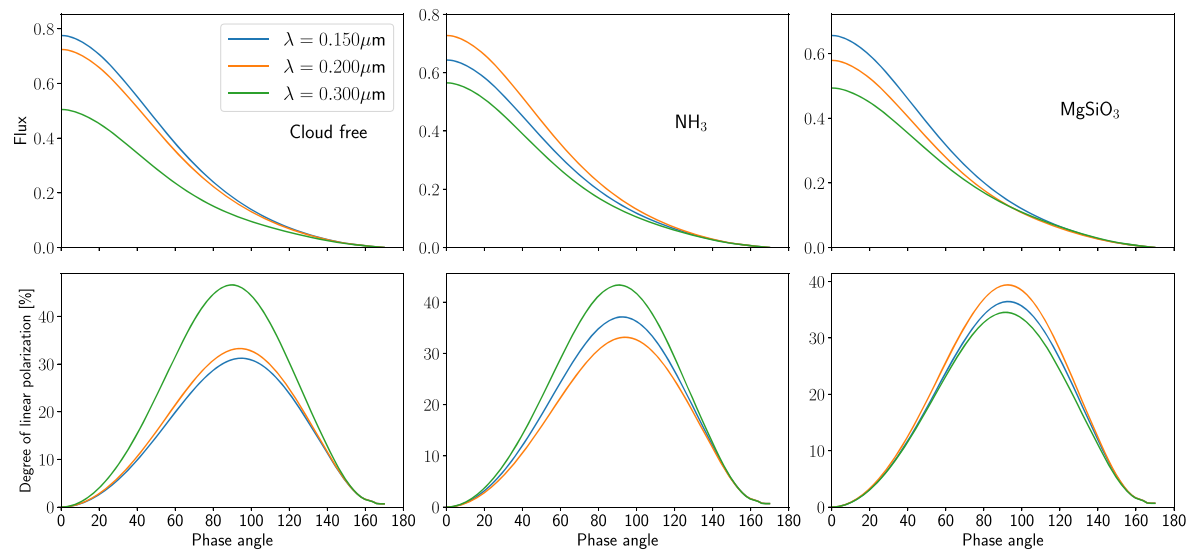

Fig. 3 Flux (top) and degree of polarization (P; bottom) as a function of phase angle at three UV wavelengths for a HD189733-like exoplanet hosting a clear atmosphere (left) and an atmosphere dominated by $\mathrm{NH}_{3}$ (middle) or $\mathrm{MgSiO}_{3}$ (right) aerosols 
signal from other contributions (e.g., interstellar medium). It may also prove useful to remove the effect of systematics provided that their signal is either a smooth function of wavelength or mimics the stellar spectrum without shifts in wavelength. In other words, dispersing the polarized light provides a natural way of separating the planet's signal (which is Doppler shifted) from other polarization signals introduced in the photon path. Cross-correlating high-resolution polarimetric spectra requires knowledge of the opacities of the molecules of interest at high resolution. This information is already available for several species relevant for exoplanet studies, particularly at optical and infrared wavelengths, but many are still lacking in the ultraviolet (e.g., $\mathrm{O}_{2}, \mathrm{O}_{3}$ ), where the polarization signal is typically strongest. However, work in this direction is being carried out and the next few years will see major improvements in this respect.

\subsection{Polarimetry in the visible/near-infrared}

In the visible and infrared part of the spectrum, polarimetry can be used to characterize efficiently the properties and distribution of aerosols and clouds in an atmosphere. Whereas polarization in the ultraviolet is more sensitive to gas and small scatterers, in the visible and infrared the contribution of aerosols becomes more important. discintegrated observations of Venus have been used by [10] to retrieve the microphysical properties (size and refractive indices) of the clouds, leading to the identification of sulfuric acid as the main constituent. On Earth, measurements of the polarization inside some absorption bands have been shown to be a powerful tool to measure cloud-top altitude and determine the vertical structure of the atmosphere [2, 28]. Dedicated studies have shown that the polarization inside absorption bands can also be used for exoplanets [5].

In the continuum, spherical scatterers can produce features such as glories and rainbows, which provide a direct way to retrieve the radius and refractive index of the scattering particles. Importantly, the visible range is the region in which the transition between a polarization strongly influenced by the gas and that more sensitive to aerosols occurs. This means that variations in both the vertical and horizontal distribution of aerosols in an atmosphere can be detected by polarimetry. In particular, polarimetry is much more sensitive to the horizontal distribution of clouds, in a way that is detectable even when the planet is not resolved [19].

The complementarity between the UV and VIS/IR can therefore provide strong constraints on the respective contributions of gas and aerosols.

\section{Instrument requirements}

With the currently available technology and what is foreseeable in the near future, spectropolarimetric observations aiming at characterizing close-in, unresolved planets would be realistically possible only for planets larger than Neptune. Smaller planets would require a prohibitive number of observations to achieve an acceptable signal-to-noise ratio, demanding unrealistic stability requirements over long timescales. To make best use of the available data analysis techniques, and thus 
to enable measurements at both high and low resolution, the ideal spectropolarimeter would have a resolution greater than 100,000: [8] shows that lower spectral resolutions disentangle the planetary and non-planetary polarization signals less efficiently. A high resolving power is therefore critical to take advantage of cross-correlation techniques, still allowing measurements at low resolution. An efficiency of at least $50 \%$ and a high stability would be also desirable. Physical considerations (e.g., Rayleigh scattering) suggest that the near-UV (170-350 nm) and the blue part of the optical waveband are valuable regions to cover for polarimetric observations. Therefore, the ideal instrument would cover these regions, possibly extending to the optical and near-infrared bands to exploit the cross-correlation technique on the wealth of molecules present at those wavelengths. The need to cover the near-UV wavelength band and the required high stability, given also by the high spectral resolution, imply that the instrument would be working in space.

Furthermore, the space-based platform gives freedom on the scheduling of the observations, which is an important point for exoplanet observations that need to be conducted along the planetary orbital phase and possibly close in time to minimize systematic uncertainties. To identify the aperture size of the telescope that is required to characterize the atmospheres of short-period ( $\simeq 2$ days) exoplanets, we computed the signal-to-noise ratio in the $250-400 \mathrm{~nm}$ spectral region as a function of telescope aperture obtained over 3 hours of shutter time and with $5 \mathrm{~nm}$ binning. We employed a telescope-spectropolarimeter system providing an average efficiency of 50\%, which is reasonable considering the available technology. Figure 4 presents the results considering G2V, F5V, and A0V host stars. By focusing on the near-UV,

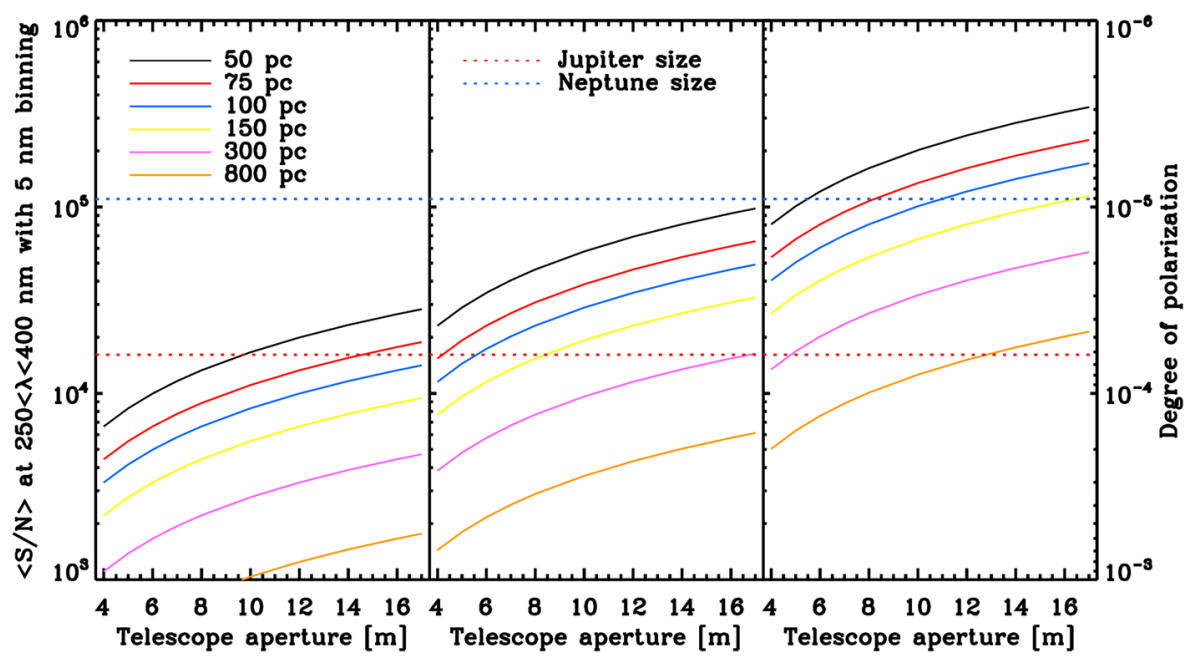

Fig. 4 Average signal-to-noise ratio of the data (i.e., inverse of the measurable polarization level) between $250 \mathrm{~nm}$ and $400 \mathrm{~nm}$ obtained with 3 hours of shutter time and $5 \mathrm{~nm}$ binning as a function of the distance to the target star (line color) and size of the telescope primary mirror. Results are for a G2V (left), F5V (center), and A0V (right) star. The red and blue dotted horizontal lines indicate the maximum degree of polarization $(\mathrm{P})$ calculated respectively for a Jupiter-radius and Neptune-radius planet in a 2-day orbit around the host star. We considered that each planet gives a maximum $\mathrm{P}$ of $30 \%$ and that the efficiency of the telescope-instrument system is on average 50\% (Figure from [6]) 
Fig. 4 shows that detecting and characterizing the atmospheres of close-in giant planets orbiting G-type stars would require a telescope with an aperture of the order of 9-10 m, or larger. Because of the increasing fluxes of the host stars, measuring polarization becomes easier with increasing the temperature of the host stars. Figure 4 clearly shows that close-in gas giants orbiting A-type stars would be excellent targets for near-UV observations. By moving towards shorter wavelengths, later spectral types would then be favored. Furthermore, a 10-m class telescope would enable the detection the polarized reflected light even for systems as far as $500 \mathrm{pc}$.

This corresponds to an L-class mission composed of a large telescope, covering from the UV to the near-IR, with full wavelength coverage, high spectral resolution, and coronagraphic (internal or external) capabilities.

Thanks to the unique possibility given by polarimetry to characterize the atmospheres also of non-transiting exoplanets and by the large number of nearby systems discovered so far by both radial velocity and transit surveys, several targets would be already available for in-depth characterization. As of today, a target list composed of planets with masses larger than Saturn (to ensure Jupiter-like planetary radii; e.g., [11]), orbital periods shorter than 3 days, and orbiting stars hotter than the Sun and closer than 200 pc would comprise about 20 systems. A number of additional (possibly better) targets can be expected to become available soon following the results of the Gaia, TESS, and PLATO missions and radial velocity surveys.

Open Access This article is licensed under a Creative Commons Attribution 4.0 International License, which permits use, sharing, adaptation, distribution and reproduction in any medium or format, as long as you give appropriate credit to the original author(s) and the source, provide a link to the Creative Commons licence, and indicate if changes were made. The images or other third party material in this article are included in the article's Creative Commons licence, unless indicated otherwise in a credit line to the material. If material is not included in the article's Creative Commons licence and your intended use is not permitted by statutory regulation or exceeds the permitted use, you will need to obtain permission directly from the copyright holder. To view a copy of this licence, visit http://creativecommons.org/licenses/by/4.0/.

\section{References}

1. Bailey, J.: Rainbows, polarization, and the search for habitable planets. Astrobiology 7, 320-332 (2007). https://doi.org/10.1089/ast.2006.0039

2. Boesche, E., Stammes, P., Bennartz, R.: Aerosol influence on polarization and intensity in nearinfrared $\{\mathrm{O} 2\}$ and $\{\mathrm{CO} 2\}$ absorption bands observed from space. J. Quant. Spectrosc. Radiat. Transf. 110(3), 223-239 (2009). https://doi.org/10.1016/j.jqsrt.2008.09.019. http://www.sciencedirect.com/ science/article/pii/S0022407308002008

3. Cotton, D.V., Bailey, J., Kedziora-Chudczer, L., Bott, K., Lucas, P., Hough, J., Marshall, J.P.: The linear polarization of southern bright stars measured at the parts-per-million level. Mon. Not. R. Astron. Soc. 455(2), 1607-1628 (2016)

4. Cotton, D.V., Marshall, J.P., Bailey, J., Kedziora-Chudczer, L., Bott, K., Marsden, S.C., Carter, B.D.: The intrinsic and interstellar broad-band linear polarization of nearby fgk dwarfs. Mon. Not. R. Astron. Soc. 467(1), 873-897 (2017)

5. Fauchez, T., Rossi, L., Stam, D.M.: The o2 a-band in the fluxes and polarization of starlight reflected by earth-like exoplanets. Astrophys. J. 842(1), 41 (2017)

6. Fossati, L., Rossi, L., Stam, D., Muñoz, A.G., Berzosa-Molina, J., Marcos-Arenal, P., Caballero, J., Cabrera, J., Chiavassa, A., Desert, J.M., et al.: Ultraviolet spectropolarimetry as a tool for understanding the diversity of exoplanetary atmospheres. arXiv:1903.05834 (2019) 
7. García Muñoz, A., Mills, F.P.: Pre-conditioned backward Monte Carlo solutions to radiative transport in planetary atmospheres. Fundamentals: Sampling of propagation directions in polarising media. A\&A 573, A72 (2015). https://doi.org/10.1051/0004-6361/201424042

8. García Muñoz, A.: On mapping exoplanet atmospheres with high-dispersion spectro-polarimetry: Some model predictions. Astrophys. J. 854(2), 108 (2018)

9. Goloub, P., Arino, O.: Verification of the consistency of polder aerosol index over land with atsr-2/ers2 fire product. Geophys. Res. Lett. 27(6), 899-902 (2000)

10. Hansen, J.E., Hovenier, J.W.: Interpretation of the polarization of Venus. J. Atmos. Sci. 31, 1137-1160 (1974). https://doi.org/10.1175/1520-0469(1974)031<1137:IOTPOV>2.0.CO;2

11. Hatzes, A.P., Rauer, H.: A definition for giant planets based on the mass-density relationship. Astrophys. J. Lett. 810(2), L25 (2015)

12. Hough, J.H., Lucas, P.W., Bailey, J.A., Tamura, M.: High-sensitivity polarimeter for the direct detection and characterization of extra-solar planets. In: Polarimetry in Astronomy, International Society for Optics and Photonics, vol. 4843, pp. 517-523 (2003)

13. Karalidi, T., Stam, D.M.: Modeled flux and polarization signals of horizontally inhomogeneous exoplanets applied to earth-like planets. A\&A 546, A56 (2012). https://doi.org/10.1051/0004-6361/ 201219297

14. Karalidi, T., Stam, D.M., Hovenier, J.W.: Flux and polarisation spectra of water clouds on exoplanets. A\&A 530, A69 (2011). https://doi.org/10.1051/0004-6361/201116449

15. Karalidi, T., Stam, D.M., Hovenier, J.W.: Looking for the rainbow on exoplanets covered by liquid and icy water clouds. A\&A 548, A90 (2012). https://doi.org/10.1051/0004-6361/201220245

16. Kemp, J.C., Henson, G.D., Steiner, C.T., Powell, E.R.: The optical polarization of the sun measured at a sensitivity of parts in ten million. Nature 326, 270-273 (1987). https://doi.org/10.1038/326270a0

17. Laven, P.: Effects of refractive index on glories. Appl. Opt. 47(34), H133-H142 (2008). https://doi.org/10.1364/AO.47.00H133, http://ao.osa.org/abstract.cfm?URI=ao-47-34-H133

18. Miles-Páez, P., Pallé, E., Osorio, M.Z.: Simultaneous optical and near-infrared linear spectropolarimetry of the earthshine. Astron. Astrophys. 562, L5 (2014)

19. Rossi, L., Stam, D.M.: Using polarimetry to retrieve the cloud coverage of Earth-like exoplanets. A\&A 607, A57 (2017). https://doi.org/10.1051/0004-6361/201730586

20. Rossi, L., Stam, D.M.: Circular polarization signals of cloudy (exo)planets. A\&A 616, A117 (2018). https://doi.org/10.1051/0004-6361/201832619

21. Rossi, L., Marcq, E., Montmessin, F., Fedorova, A., Stam, D., Bertaux, J.L., Korablev, O.: Preliminary study of venus cloud layers with polarimetric data from spicav/vex. Planet. Space Sci. 113 - 114(0), 159-168 (2015). https://doi.org/10.1016/j.pss.2014.11.011, sI:Exploration of Venus

22. Rossi, L., Berzosa-Molina, J., Stam, D.M.: Pymiedap: a python-fortran tool for computing fluxes and polarization signals of (exo)planets. A\&A 616, A147 (2018). https://doi.org/10.1051/0004-6361/ 201832859

23. Saar, S.H., Seager, S.: Uses of linear polarization as a probe of extrasolar planet atmospheres. In: Deming, D., Seager, S. (eds.) Scientific Frontiers in Research on Extrasolar Planets, Astronomical Society of the Pacific Conference Series, vol. 294, pp. 529-534 (2003). arXiv:astro-ph/0305436

24. Seager, S., Whitney, B., Sasselov, D.: Photometric light curves and polarization of close-in extrasolar giant planets. Astrophys. J. 540(1), 504 (2000)

25. Shalygina, O.S., Korokhin, V.V., Starukhina, L.V., Shalygin, E.V., Marchenko, G.P., Velikodsky, Y.I., Starodubtseva, O.M., Akimov, L.A.: The north-south asymmetry of polarization of Jupiter: The causes of seasonal variations. Sol. Syst. Res. 42, 8-17 (2008). https://doi.org/10.1134/S0038094608010024

26. Sing, D.K., Fortney, J.J., Nikolov, N., Wakeford, H.R., Kataria, T., Evans, T.M., Aigrain, S., Ballester, G.E., Burrows, A.S., Deming, D., Désert, J.M., Gibson, N.P., Henry, G.W., Huitson, C.M., Knutson, H.A., Lecavelier, D.es.E.tangs.A., Pont, F., Showman, A.P., Vidal-Madjar, A., Williamson, M.H., Wilson, P.A.: A continuum from clear to cloudy hot-Jupiter exoplanets without primordial water depletion. Nature 529(7584), 59-62 (2016). https://doi.org/10.1038/nature16068, arXiv:1512.04341

27. Stam, D.M.: Spectropolarimetric signatures of Earth-like extrasolar planets. A\&A 482, 989-1007 (2008). https://doi.org/10.1051/0004-6361:20078358, arXiv:0707.3905

28. Stam, D.M., De Haan, J.F., Hovenier, J.W., Stammes, P.: Degree of linear polarization of light emerging from the cloudless atmosphere in the oxygen A band. J Geophys Res 104, 16843-16858 (1999). https://doi.org/10.1029/1999JD900159

29. Stam, D.M., Hovenier, J., Waters, R.: Polarization of extrasolar planets: sample simulations. In: Deming, D., Seager, S. (eds.) Scientific Frontiers in Research on Extrasolar Planets, Astronomical Society of the Pacific Conference Series, vol. 294, pp. 535-538 (2003) 
30. Stam, D.M., Hovenier, J.W., Waters, L.B.F.M.: Using polarimetry to detect and characterize Jupiterlike extrasolar planets. A\&A 428, 663-672 (2004). https://doi.org/10.1051/0004-6361:20041578

31. Stam, D.M., de Rooij, W.A., Cornet, G., Hovenier, J.W.: Integrating polarized light over a planetary disk applied to starlight reflected by extrasolar planets. A\&A 452, 669-683 (2006). https://doi.org/10. 1051/0004-6361:20054364

32. Tomasko, M.G., Smith, P.H.: Photometry and polarimetry of Titan - Pioneer 11 observations and their implications for aerosol properties. Icarus 51, 65-95 (1982). https://doi.org/10.1016/0019-1035(82) 90030-6

Publisher's note Springer Nature remains neutral with regard to jurisdictional claims in published maps and institutional affiliations. 